\title{
Developing Finite Element Simulation of Sheet Metal Stamping Processes - a contribution to Virtual Manufacturing
}

\author{
A.D. Santos 1,2, P. Montel 1, J.F. Duarte 1,2, A.Barata da Rocha 1,2 \\ 1 INEGI/CETECOP - Institute of Mech. Engineering and Industrial \\ Management \\ Rua do Barroco, 174/214, S.Mamede Infesta, Portugal \\ 2 DEMEGI - Department of Mechanical Engineering of the University \\ of Porto
}

Rua dos Bragas, 4099 Porto Codex, Portugal

\begin{abstract}
Finite Element Modeling (FEM) can be a valuable addition to computer integrated manufacturing (CIM) along with other kinds of analysis and tools such as computer aided design (CAD), manufacturing (CAM) and engineering (CAE).

A FEM program for analyzing sheet stamping operations has been developed to simulate sheet metal stamping processes. Most of these processes involve axi-symmetric, plane strain or plane stress analysis for which a two-dimensional finite element simulation code can give important and necessary directions to the best compromise of stamping variables to be used, before tool manufacturing and parts production. Major advantages of such analysis are fast answers and possible use of PC's instead of workstations.

This paper describes the use of a static explicit finite element simulation code and its implementation to work and be used on a Pentium based PC. Comparison of simulated results and experimental ones is presented, as well as comparison of CPU time efficiency for PC and workstation.
\end{abstract}

FEM, CAD, CAE, CIM

Keywords 


\section{INTRODUCTION}

Simulation of sheet metal stamping processes by using Finite Element Method has been proving to be a useful and reliable aid to help tool designers to make right decisions before tool manufacturing and trial production, thus saving time and cost [2]. However, some difficulties must be overcome in order to obtain such goals. Concerning sheet metal forming processes the main problems to overcome in simulation include an efficient approach to describe tool geometry [Santos, 1992], the treatment of tool-work contact [Makinouchi, 1990(1)] in order to deal with the highly deformation dependent contact problems with friction, and also the robustness of the code. From these variables the robustness of the code plays an important role, since sheet forming simulation is prone to instabilities due to the non-linearity characteristic of these processes [Saracibar, 1990]. Many elasto-plastic finite element codes employ the implicit time integration scheme, which has the advantage of large time increments and satisfies rigorously the equilibrium at the end of time step [Makinouchi, 1990(2)]. However sometimes calculations stop due to lack of convergence. On the contrary the explicit integration scheme restricts the time increment to a very small size in order to maintain out of balance forces within admissible tolerances and this scheme has the important advantage of a stable and robust solution. The lack of convergence for implicit analysis is more evident when 3-dimensional forming problems are simulated [Rebelo, 1992], due to additional instabilities. The robustness of the explicit analysis is the main reason why the code herein presented uses such formulation.

Another important concern when developing a code is its user friendliness and its compromise with speed of calculation. The existent growing development of fast processors for personal computers is beginning to make possible the use of PC's to simulate sheet metal forming with the advantages of using common, non-expensive computers with user friendly interfaces.

This paper presents the implementation of 2-dimensional code to be used in a personal computer including the development and the use of standard PC tools for pre and postprocessing. Comparison of simulation and experimental results are performed to test the accuracy and reliability of the explicit code and comparison of CPU time is performed between PC and workstation in order to test the efficiency and cost effectiveness of this solution.

\section{FEM CODE}

The simulation results were obtained by using ITAS code. This is a Japanese FEM code developed by Makinouchi and co-workers [Makinouchi, 1990(1)].

\subsection{Variational principle}

The updated Lagrangian rate formulation is the base of the incremental elasto-plastic finite element code. The rate form of equilibrium equations and boundary conditions are described by the principle of virtual velocity, as it is proposed by McMeeking and Rice [Mc Meeking, 1975], on the form: 


$$
\int_{V}\left\{\left(\dot{\sigma}_{i j}-2 \cdot \sigma_{i k} \cdot D_{k j}\right) \cdot \delta D_{i j}+\sigma_{j k} \cdot L_{i k} \cdot \delta L_{i j}\right\} \cdot d V=\int_{s_{t}} \dot{t}_{i} \cdot \delta v_{i} \cdot d S
$$

Here, $\delta \underset{\sim}{v}$ is the virtual velocity field satisfying the boundary condition $\delta \underset{\sim}{v}=0$ on $S_{t} . V$ and $S$ denote the region-occupied, at time $t$, by body and its boundary, respectively. $S_{t}$ is the part of the boundary $S$, on which the rate of the nominal traction vector, $\underset{\sim}{t}$ is prescribed. $\underset{\sim}{\sigma}$ denotes the

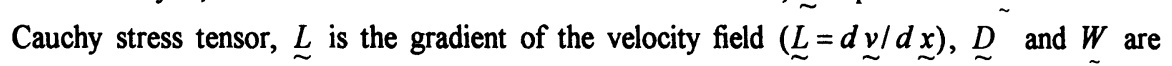
respectively, the symmetric and anti-symmetric parts of $\underset{\sim}{L}$, and $\dot{\sigma}_{i j}=\dot{\sigma}_{i j}-W_{i k} \cdot \sigma_{k j}+\sigma_{i k} \cdot W_{k j}$ represents the Jaumann derivative of $\underset{\sim}{\sigma}$.

\subsection{Constitutive equation}

The small strain isotropic linear elasticity and the large deformation rate-independent workhardening plasticity is assumed. In order to deal with anisotropy of sheet metal, Hill's quadratic yield function and the associative flow law are used. Following Cao and Teodosiu [Cao, 1989], the constitutive equation of the present model is written in the form:

$$
\dot{\sigma}_{i j}=C_{i j k l}^{e p} \cdot D_{k l}=C_{i j k l}^{e p} \cdot L_{k l}
$$

where the constitutive matrix $C_{i j k l}^{e p}$ is:

$$
C_{i j k l}^{e p}=\lambda \delta_{i j} \delta_{k l}+\mu\left(\delta_{i k} \delta_{j l}+\delta_{l l} \delta_{j k}\right)-\alpha \cdot f_{0} Z_{i j} Z_{k l}
$$

In this equation $\lambda$ and $\mu$ are the Lamé elastic constants, $\delta_{i j}$ is Kronecker's delta and $\alpha$ is a constant; $\alpha=1$ for plastic loading and $\alpha=0$ for elastic state and unloading. $f_{0}$ and $Z_{i j}$ are expressed by:

$$
\begin{aligned}
& f_{0}=\frac{2 \mu}{Z_{i j} Z_{k l}+Z_{i j} Z_{k l}+H_{0} /(2 \mu)} \\
& Z_{i j}=M_{i j r s} \sigma_{r s}^{\prime}
\end{aligned}
$$

Here $H_{0}$ is the work-hardening modulus, $\sigma_{i j}^{\prime}$ is the stress deviator and $M_{i j k}$ denote Hill's anisotropic parameter, assuming that sheet remains orthotropic during deformation, while the orthotropic axis are subjected to a time-dependent rotation $R_{i j}$. The explicit form of $M_{j j k}$ is written as:

$$
\bar{\sigma}^{2}=M_{i j r s}^{0} \cdot \sigma_{i j} \cdot \sigma_{r s}
$$




$$
=F\left(\sigma_{22}-\sigma_{33}\right)^{2}+G\left(\sigma_{33}-\sigma_{11}\right)^{2}+H\left(\sigma_{11}-\sigma_{22}\right)^{2}+2 L \sigma_{23}^{2}+2 M \sigma_{13}^{2}+2 N \sigma_{12}^{2}
$$

where $\mathrm{F}, \mathrm{G}, \mathrm{H}, \mathrm{L}, \mathrm{M}, \mathrm{N}$ are material parameters; assuming that their value is kept constant during deformation, thus the evolution of $M_{i j k}$ is given by:

$$
M_{i j s}=R_{i m} \cdot R_{j n} \cdot R_{r p} \cdot R_{s q} \cdot M_{m n p q}^{0}
$$

\subsection{Finite Element Discretization}

Equation (2) corresponds to an inviscid behaviour, since it is insensitive to the choice of the time scale. Thus, the time increment can be replaced by the increment of any other monotonously increasing parameter, e.g. an imposed displacement. Performing a standard finite element discretization, namely dividing the sheet into finite elements and introducing the expression of the incremental displacements in terms of the shape functions into the incremental forms of (1) and (2) yields a system of algebraic equations of matrix form:

$$
[K] \cdot\{d u\}=\{d f\}
$$

where $\{d u\}$ is the vector of nodal displacements increment and $\{d f\}$ the vector of nodal forces increment, corresponding to a trial increment $d w$ of the loading parameter, while $[K]$ is the symmetric elasto-plastic tangent stiffness matrix [Makinouchi, 1985].

ITAS code uses an explicit approach to obtain the solution of Eq.(8). The stiffness matrix $[K]$ is described at time $t$ and it is regarded constant within the time increment $\Delta t$. The socalled Rmin method [Yamada, 1968; Makinouchi, 1990(2)] is used to impose limitation to the time step in such a way that no significant changes in the stiffness matrix occur during the increment. This method assures the accuracy of the explicit integration scheme and it is used to choose the size of the increment. This size is calculated assuming that, during one increment:

(I) - a finite element can not change from elastic to plastic state and vice versa

(II) - the largest value of the incremental principal strains attains a limiting value of $\Delta \varepsilon_{\max }=0.002$

(III)- the largest absolute value of the incremental rotations attains a limiting value of $\Delta \theta_{\max }=0.5^{\circ}$

(IV) - a free node will not come into contact

(V) - a contacting node will not get free

(VI) - a sticking node will not slide

(VII)- a sliding node will not stick.

The last 4 conditions are concerned with boundary conditions (contact and sliding states) and they assure that, during one incremental step, boundary conditions are kept unchanged.

The procedure to apply for Rmin strategy and calculation of the increment size involves the following steps:

- at the beginning of the increment a fictitious incremental tool displacement $\Delta a$ is prescribed;

- the stiffness equation is solved for a fictitious solution $\Delta u$, correspondent to the previous tool displacement $\Delta a$; 
- for each of the previous conditions I VII, a ratio $\mathbf{r}$ is found, between the obtained solution $\Delta u$ and a "real" solution that validates each condition; the minimum of all these ratio $r$ will be the value of Rmin;

- the obtained solution $\Delta u$ is weighted by $R \min$ coefficient $(0<R \min <1)$, the size of the increment will be $R \min * \Delta u$ and this is the value used to update the configuration of the sheet, the total displacements, the total (Cauchy) stresses and the yield limit of each element.

\subsection{Finite Elements}

The four node bilinear isoparametric element is the basic element used. This element can be used with different stress integration methods: the full integration, the reduced integration, the selective reduced integration and the hour glass control stabilized matrix method [11]. From these integration schemes the third is the one used in the simulations herein presented, since it offers a good compromise between performance and results.

\subsection{Description of Tool Geometry}

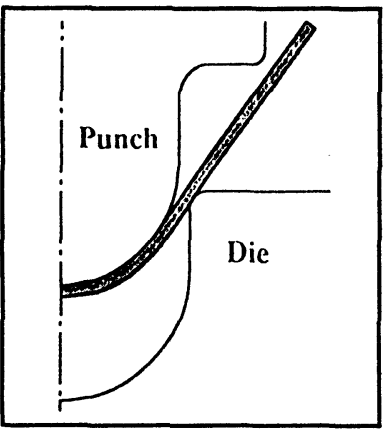

Figure 1 - Function approach

Several tool descriptions can be used to define tool geometry. They include the Point Data approach, the Mesh approach and the Parametric approach [Santos, 1993]. For 2-dimensional problems the function approach is suitable due to its easy generation and complete potential for tool description. In this approach the geometry of tool surface is expressed by a combination of subdomains which are represented by elementary functions of the form:

$$
f(x, a, c)=0 \text { in domain } \mathrm{D}
$$

In this equation $a$ and $c$ are constants which define shape and position of the curve. For 2D situation straight lines and arcs are used and explicit form of Eq.(10) are given by:

$$
\begin{aligned}
& y=c . x+a \quad \text { for } \quad x_{A} \leq x \leq x_{B} \\
& \left(x-a_{1}\right)^{2}+\left(y-a_{2}\right)^{2}=c \quad \text { for } \theta_{A} \leq \theta \leq \theta_{B}
\end{aligned}
$$

where $\theta$ is the angle for the arc of circle.

Motion of the tool is given by an incremental rigid motion $\Delta a$ as

$$
f(x, a+\Delta a, c)=0
$$

Tool is modeled by considering its boundaries as being rigid. 


\subsection{Pre and Post-processing}

In order to use a PC to run a simulation code for sheet metal forming it is important also to have or develop pre and post processing capabilities that can be used on the same computer. In our case we are using an widespread CAD software (AutoCad) for pre-processing the shape of tools to be used in simulation. Such data is written into a file in DXF format and read by the code at the same time as the sheet data, material data and other variables (Figure 2). Concerning post-processing, AutoCad is used to output sheet geometry as well as contacting nodes and its force vectors (Figure 2). To output stress and strain distribution we are using a visualization code developed at our Department of Mechanical Engineering of Porto University.

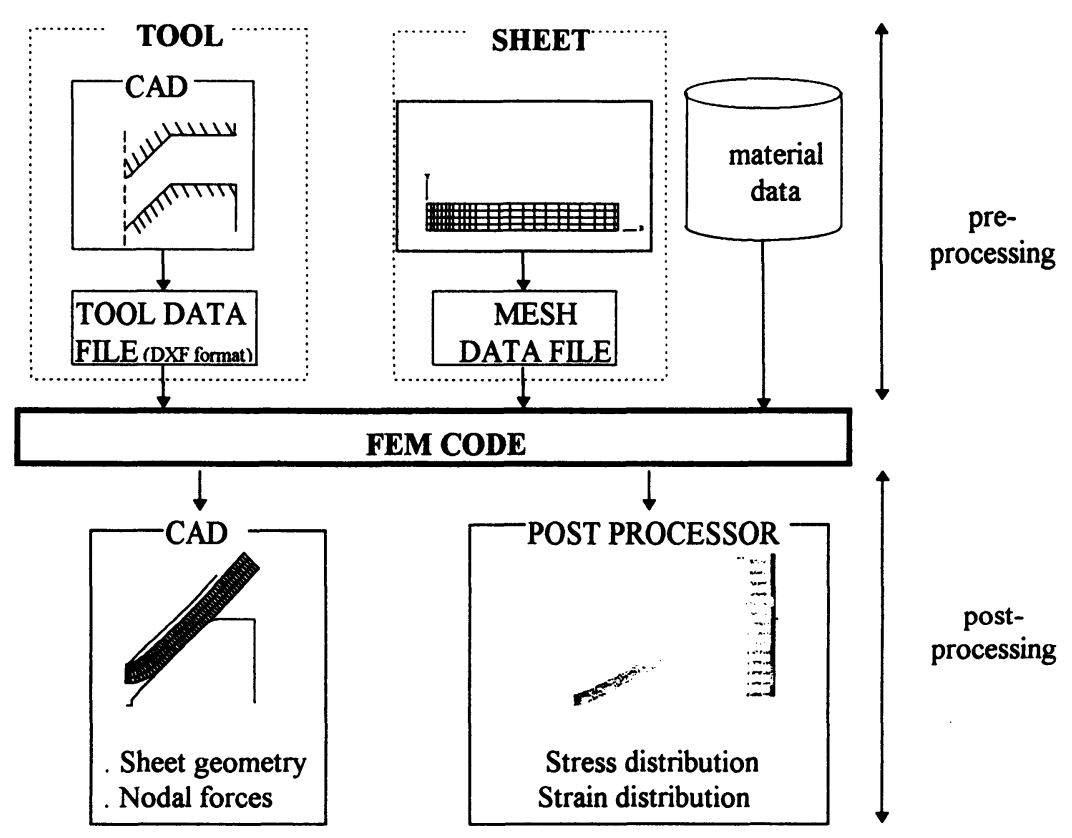

Figure 2 - pre/post processing organization.

\section{RESULTS}

In order to test the accuracy and reliability of the code, two test examples have been simulated and comparison with experiments have been performed. The tests were a V-bending example and a sheet bending example with a cylindrical punch. 


\subsection{V-bending}

This example uses a standard tool from a press brake machine. Tool geometry as well as comparison of simulated and experimental geometries are presented in Figure 3 for three stages of the process $(10,15$ and $20 \mathrm{~mm}$ punch displacement). Material is mild steel with $3 \mathrm{~mm}$ thickness, and sheet is discretized by a mesh of 90 elements (only $1 / 2$ of the geometry is used for calculation, due to symmetry). Punch reaches the $20 \mathrm{~mm}$ displacement after 690 steps. As seen, there is a good agreement between simulated and experimental deformed geometry.

\subsection{Sheet bending with a cylindrical punch}

This test uses the same standard tool but now with a cylindrical punch. Sheet characteristics are the same as in the previous example. Calculated and experimental geometries are presented in Figure 4 for the same stages as the V-bending process. The deformed geometry obtained by simulation agrees quite well with experiment also for this example.

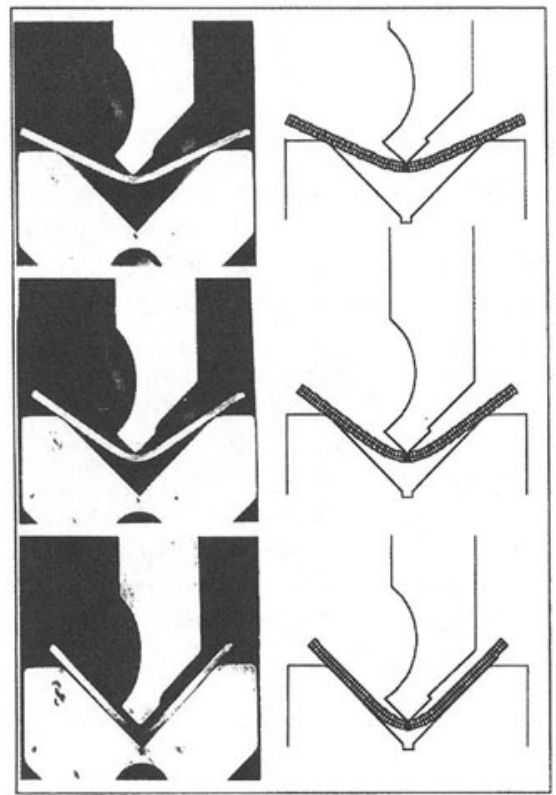

Figure 3 - compared geometries for V-bending

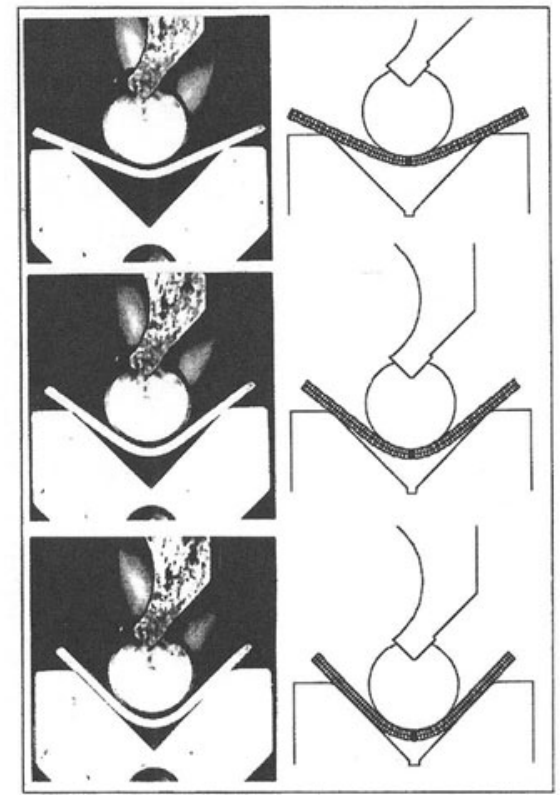

Figure 4 - compared geometries for cylindrical punch bending. 


\subsection{CPU time comparison}

Time comparison between PC with a Intel Pentium processor running at $90 \mathrm{MHz}$ and a $\mathrm{HP}$ workstation model 720 was performed to test the efficiency of this implementation. Figure 5 shows comparison time results for calculation of the $\mathrm{V}$ bending process example, with and without time optimization during code compilation.

To reach the $20 \mathrm{~mm}$ punch displacement, maximum time reported as $100 \%$ in Figure 5 is $6 \mathrm{mn} \mathrm{14s}$. This time corresponds to workstation CPU time without optimization. As it can be seen

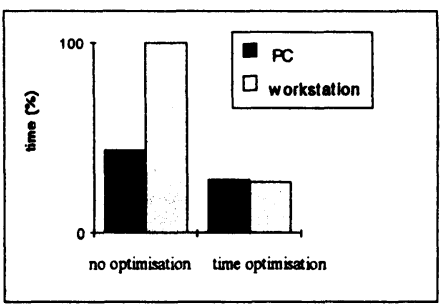

Figure 5 - CPU time comparison the PC computation time is very close to the workstation computation time (with optimization), which shows the great potential of using a PC to perform this kind of simulation.

\section{CONCLUSIONS}

An integrated system for analysis of 2D sheet metal forming processes has been presented. Until recently such system could only work by using workstations, specially due to the processing time needed for the FE code. It is shown that with actual processing power of PC's this task can be accomplished by using this kind of computers and in order to work efficiently it has been developed and presented pre and post-processing capabilities.

The most important part of this system is the FE code. The explicit formulation of the presented code gives the necessary robustness to overcome all non-linearities and difficulties of this kind of processes. Two simulation examples have been presented and comparison between simulation and experiment has shown a good agreement of the results.

It is expected that this type of implementation and its further development can contribute for the user friendliness and efficiency of Finite Element Simulation, which is nowadays a proved "tool" to be used in the design of sheet metal components, allowing us to predict forming defects that may appear during production, thus eliminating or reducing the trial and error stage after tool fabrication and before parts production.

\section{ACKNOWLEDGMENTS}

The authors wish to thank the financial support provided by Junta Nacional de Investigação Científica e Tecnológica of Portugal (JNICT), through project PBIC/TPR/2557/95. Also they would like to express their gratitude to Dr.A.Makinouchi and Sheet Forming Simulation Research Group of Japan for the availability of ITAS code and the opportunities and fruitful discussions during the stay at Riken Institute of Japan, of one of the authors. The authors are also grateful to Mr.Hipólito Reis, Mr.Fernando Junqueira and Mr.António J.Gomes for their help during experiments. 


\section{REFERENCES}

Cao H.L., Teodosiu C. (1989) "Finite element calculation of springback effects and residual stresses after 2D deep drawing", Computational Plasticity'89, pp.959-971.

Duarte J.F., Santos A.D., Barata da Rocha A. (1995) "FE Simulation as an Aid to Design Sheet Metal Parts", SheMet'95-3. International Conference on Sheet Metal, June 26-28, University of Central England, Birmingham.

Makinouchi A.(1985) "Elasto-plastic stress analysis of bending and forming of sheet metal", Comp. Model. Sheet Metal Form. Proc., Eds. Wang N.M. \& Tang S.C., Warrendale, pp. 161-176.

Makinouchi A., Shirataki Y., Liu S.D., Nagai Y. (1990)(1) "Generalization of Tool-Work contact conditions for elasto-plastic analysis in Forming Processes", 3rd ICTP, pp.1161, Kyoto, Japan.

Makinouchi A. (1990)(2) "Contact descriptions in finite element simulation of metal forming processes, Computer aided innovation of new materials, CAMSE'90, Conference Proceedings pp.597-602, August 28-31 1990, Tokyo, Japan.

Mc Meeking R.M., Rice J.R. (1975) "Finite element formulation for problems of large elasticplastic deformation"- International Journal of Solids Structures, pp.601-616, vol.11.

Rebelo N., Nagtegaal J.C., Taylor L.M., Passman R. (1992) "Comparison of implicit and explicit finite element methods in the simulation of metal forming processes", Numiform' 92 , Conference Proceedings pp 99-108, September 14-18 1992, Valbonne, France.

Santos A., Makinouchi A. (1992) "Comparison of different approaches to describe tool geometry in FE simulation of 3D sheet stamping processes"- Computational mechanics 92 theory and application, Conference Proceedings, pp.171, Vol.1, December 17-22, 1992, Hong Kong.

Santos A., Makinouchi A. (1993) "Contact strategies to deal with different tool descriptions in static explicit FEM of 3D sheet metal forming simulation", Numisheet'93, Conference Proceedings pp 261-270, August 31-September 2 1993, Isehara, Japan.

Saracibar C. (1990) "Analisis por el metodo de los elementos finitos de procesos de conformado de laminas metalicas", Dissertation, Politechnique University of Catalunya, Barcelona, Spain.

Takizawa H., Makinouchi A., Santos A., Mori N. (1991) "Simulation of 3-D sheet bending processes", FE-simulation of 3-D sheet metal forming processes in automotive industry, VDI berichte 894, Conference Proceedings pp 167-184, May 14-16 1991, Zurich.

Yamada Y., Yoshimura N., Sakurai T. (1968) International Journal of Mechanical Science, 10, 343-354. 


\section{BIOGRAPHY}

Abel D. dos Santos is Assistant Professor of Faculty of Engineering of Porto University and responsible for Section of Materials and Technological Processes of the Department of Mechanical Engineering and Industrial Management. He is also Assistant Researcher at INEGI Institute of Mechanical Engineering and Industrial Management - where it is mainly involved with Sheet Metal Forming Simulation.

He has been graduated at the Department of Mechanical Engineering of Porto University and obtained his Msc. degree in Structural Mechanics at the same University. His $\mathrm{PhD}$ degree has been obtained at Tokyo University, Japan, being supervised by Prof. T.Nakagawa of Tokyo University and A.Makinouchi of Materials Fabrication Laboratory of Riken Institute. His PhD work has been mainly concerned with development of a Finite Element code for Sheet Metal Forming Simulation intended to be applied mainly in the automobile industry. 